\title{
Mid-infrared spectroscopy and multivariate analysis for determination of tetracycline residues in cow's milk
}

\author{
Lizeth Mariel Casarrubias-Torres ${ }^{1}$, Ofelia Gabriela Meza-Márquez², \\ Guillermo Osorio-Revilla², Tzayhrí Gallardo-Velazquez ${ }^{1}$
}

Instituto Politécnico Nacional, ${ }^{1}$ Escuela Nacional de Ciencias Biológicas - Santo Tomás, Department of Biophysics, ${ }^{2}$ Escuela Nacional de Ciencias Biológicas Zacatenco, Department of Biochemical Engineering, City of Mexico, Mexico

Received June 24, 2017

Accepted May 15, 2018

\begin{abstract}
Mid-infrared spectroscopy and chemometric analysis were tested to determine tetracycline's residues in cow's milk. Cow's milk samples $(n=30)$ were spiked with tetracycline, chlortetracycline, and oxytetracycline in the range of $10-400 \mu \mathrm{g} / 1$. Chemometric models to quantify each of the tetracycline's residues were developed by applying Partial Components Regression and Partial Least Squares algorithms. The Soft Independent Modeling of Class Analogy model was used to differentiate between pure milk and milk sample with tetracycline residues. The best models for predicting the levels of these antibiotics were obtained using Partial Least Square 1 algorithm (coefficient of determination between 0.997-0.999 and the standard error of calibration from 1.81 to 2.95). The Soft Independent Modeling of Class Analogy model showed well-separated groups allowing classification of milk samples and milk sample with antibiotics. The obtained results demonstrate the great analytical potential of chemometrics coupled with mid-infrared spectroscopy for the prediction of antibiotic in cow's milk at a concentration of microgram per litre $(\mu \mathrm{g} / \mathrm{l})$. This technique can be used to verify the safety of the milk rapidly and reliably.
\end{abstract}

Antibiotics, human health, multivariate analysis, chemometrics, vibrational spectral data

The tetracyclines (TCs) have a wide antibacterial range and bacteriostatic action, and a good activity against infections caused by a number of bacteria. The TCs are permitted in a diversity of food-producing animals (sheep, poultry, pigs, cattle and fish) (Debuf 1998). The usage of TCs in dairy husbandry and the failure to follow good veterinary practices can lead to unsafe residue concentrations in various tissues and milk, with potential adverse effects on human health (for example allergic reactions in some hypersensitive individuals). Also the TCs residues may affect aquatic ecosystems (Feng-Jiao et al. 2014).

To guarantee high quality of human food, several regulatory authorities have defined the maximum residue limits (MRLs) allowing only trace amounts of residues of veterinary drugs in food for human intake. The MRLs are based on the acceptable daily intake of each drug; maximum food intake is also taken into account. The MRLs are established at $\mathrm{mg} \cdot \mathrm{kg}^{-1}$ or $\mu \mathrm{g} \cdot \mathrm{kg}^{-1}$. The US Food and Drug Administration (FDA) has set tolerances for the residues of tetracycline, chlortetracycline and oxytetracycline in bovine milk at $300 \mu \mathrm{g} \cdot \mathrm{l}^{-1}$ (Riviere et al. 2003), whereas in the European Union, the MRL is established at $100 \mu \mathrm{g} \cdot \mathrm{kg}^{-1}$ (Commission Regulation (EU) No. 37/2010).

Avoidance of TC residues is an important focus of the dairy industry. Judicious use of antibiotics, followed by an appropriate milk withholding time and screening for residues are essential aspects of milk quality management for assuring human food safety (Boeckman and Carlson 2006). Consequently, it is important to develop analytical procedures capable of determining the levels of TCs and to evaluate their presence in milk in order to protect human health (Casella and Picerno 2009).

Address for correspondence:

Tzayhrí Gallardo-Velazquez

Instituto Politécnico Nacional

Prolongación de Carpio y Plan de Ayala S/N. Col. Santo Tomás

C.P. 11340. Ciudad de México, México
Phone: +015557296000 ext. 62305

E-mail: gtzayhri@yahoo.com

http://actavet.vfu.cz/ 
A number of analytical procedures have been widely used for the quantification of TCs in real matrixes. These include: microbiological assay (Nagel et al. 2011), differential scanning calorimetry (DSC) (Yildiz and Unluturk 2009), capillary electrophoresis (CE) (Ibarra et al. 2011), enzyme-linked immunosorbent assays (ELISA), radioimmunoassays, biosensors and chromatographic techniques, such as high-performance liquid chromatography (HPLC), coupled with different detection systems (Toldrá and Reig 2006), liquid chromatography operating under reverse phase mode (Aderson et al. 2005) and coupled with several detection schemes such as spectrophotometry (Viñas et al. 2004; Andersen et al. 2005; Fritz and Zuo 2007), electrochemical (Casella and Picerno 2009), fluorescence (Pena et al. 2005; Pena et al. 2007; Schneider et al. 2007), or mass spectrometry (Andersen et al. 2005; Pena et al. 2007), among others.

The above analytical methods have proven useful in evaluating the concentrations of TCs in milk. Nevertheless, these techniques are difficult, laborious, requiring a significant investment in analysis time and skilled personnel (Toldrá and Reig 2006). Currently, Fourier transform mid-infrared (FTIR) spectroscopy is used to substitute old procedures of analysis. Mid FTIR is fast, requiring only a small sample; no sample preparation or use of solvents are necessary before the analysis.

A small number of spectroscopic applications to identify analytes at concentrations of microgram per litre $(\mu \mathrm{g} / \mathrm{l})$ have been demonstrated. Mid-infrared with chemometrics has been useful for the identification of veterinary residues at $\mu \mathrm{g} / \mathrm{l}$ concentrations (Sivakes ava and Irudayaraj 2002; Dračková et al. 2009; Meza-Márquez et al. 2011; MezaMárquez et al. 2012). Therefore, the aim of this study was use the Mid-FTIR spectroscopy with chemometric analysis to quantify tetracycline, chlortetracycline, and oxytetracycline in cow's milk at concentrations of $\mu \mathrm{g} / \mathrm{l}$.

\section{Materials and Methods}

Reagents

The tetracycline (tetracycline hydrochloride), chlortetracycline (chlortetracycline hydrochloride), and oxytetracycline (oxytetracycline hydrochloride) were purchased from Sigma-Aldrich (St Louis, Mo, USA). The chemicals were of analytical grade (J.T. Baker Co., Phillipsburg, NJ, USA).

\section{Standard solutions}

Stock standard solution ( $1 \mathrm{mg}$ per $\mathrm{ml}$ ) of each antibiotic (tetracycline, chlortetracycline, and oxytetracycline) was prepared dissolving $10 \mathrm{mg}$ in $10 \mathrm{ml}$ of water. Working solutions with different concentrations were prepared by dilution of stock solutions in water. All stock standard solutions and working standard solutions were prepared immediately before use.

Samples

Ten samples of pasteurized whole bovine milk were purchased at retail hypermarkets in Mexico City. To identify TCs in whole bovine milk, the samples were analyzed by immunosorbent assay (ELISA). RIDASCREEN kits (R-Biopharm A G, Darmstadt, Germany) were used for determination of TCs.

\section{Sample preparation}

Negative milk samples determined by ELISA were added to working solutions containing different concentrations of the corresponding antibiotic (tetracycline, chlortetracycline, and oxytetracycline) so that $1 \mathrm{ml}$ of the working solution of antibiotic was spiked to $4 \mathrm{ml}$ of milk to give the required concentration.

Thirty-five milk samples with each antibiotic (milk-tetracycline, milk-chlortetracycline, and milkoxytetracycline) within the concentration range between 10 and $400 \mu \mathrm{g} / \mathrm{l}$ were prepared. The calibration set included 30 milk samples with each antibiotic and the validation set included 15 samples (five milk samples with each antibiotic).

\section{Mid-infrared spectra acquisition}

A PerkinElmer 1600 Series FTIR spectrophotometer (PerkinElmer Inc., Massachusetts, USA) was used. The equipment included an attenuated total reflection accessory (PerkinElmer Inc., Massachusetts, USA) with a zinc selenide ( $\mathrm{ZnSe}$ ) crystal. The spectral data were recorded at $4000-550 \mathrm{~cm}^{-1}$ (resolution of $4 \mathrm{~cm}^{-1}$ ) over $64 \mathrm{scans}$ at controlled temperature $\left(23 \pm 1{ }^{\circ} \mathrm{C}\right)$ and steady humidity $(30 \%)$. After each sample, the crystal was rinsed with $\operatorname{Extran}^{\circledR}$ (Merck KGaA, Darmstadt, Germany), cleaned with water and dried with a soft tissue. 
Multivariate analysis

Development of models for quantification

Spectrum Quant+ software version 4.51 .02 (Perkin Elmer, Inc., Massachusetts, USA) was used for multivariate analysis. Partial Least Squares (PLS1 and PLS2) and Principal Component Regression (PCR) algorithms were used for multivariate analysis.

Diverse pre-treatments were tested to the Mid FTIR spectra: Savitzky-Golay filter ( 5 or 13 smoothing points); first and second derivatives (Savitzky-Golay method) and normalization (Standard Normal Variate, SNV).

The $\mathrm{R}^{2}$ (coefficient of determination), SEC (the standard error of calibration), and SEP (the standard error of prediction) were used to assess the models. Models with the smallest SEC and the highest $\mathrm{R}^{2}$ were chosen. The accuracy of the validation was assessed based on the SEP and $\mathrm{R}^{2}$.

Discrimination between milk samples and their mixtures with antibiotics (milk-tetracycline, milkchlortetracycline and milk-oxytetracycline) was done by SIMCA (Soft Independent Modeling of Class Analogy). The SIMCA model was developed with Assure I.D software version 3.0.0132 (Perkin Elmer, Inc., Massachusetts, USA), and divided into four classes: a) milk, b) milk-tetracycline, c) milk-chlortetracycline, and d) milkoxytetracycline. Thirty Mid FTIR spectra of each class (a total of 120 Mid FTIR) were used for the calibration set and 20 spectra (five of each class) for the validation set.

Different pretreatments were tested: second derivative (Savitzky-Golay method), smoothing (Savitzky-Golay filter, 13 points), ambient filters $\left(\mathrm{CO}_{2}\right.$ and $\left.\mathrm{H}_{2} \mathrm{O}\right)$ and normalization (Standard Normal Variate, SNV).

Class projections ( $99 \%$ of confidentiality) and interclass distances (separation of classes based on factor loading) were used to assess the SIMCA model.

\section{Results}

\section{Assay of TCs by ELISA}

The ELISA method presented very high correlation between TCs concentrations $(\mu \mathrm{g} / \mathrm{l})$ and maximum absorbance. The calibration curve had a $\mathrm{R}^{2}=0.93$ ( “ $y$ " = absorbance; and " $x "=$ TCs concentrations, $\mu \mathrm{g} / \mathrm{l})$. Ten samples of pasteurized whole bovine milk were analysed by ELISA. According to this assay, no residues of TCs were detected in the milk samples; hence these milk samples were used to interpret the mid-infrared spectra and to build the calibration models.

\section{Interpretation of mid-infrared spectra}

Representative mid-infrared spectrum collected from pure cow's milk is shown in Fig. 1. The bands from 3600 to $3000 \mathrm{~cm}^{-1}$ can be associated with $\mathrm{O}-\mathrm{H}$ stretching vibrations (water). At $3000-2800 \mathrm{~cm}^{-1}$ they are related to milk's fatty acid $\mathrm{CH}_{2}$ stretching. The peak between $1765-1730 \mathrm{~cm}^{-1}$ correspond to $\mathrm{C}=\mathrm{O}$ stretching modes (triglyceride). At $1650 \mathrm{~cm}^{-1}$ it is assigned to $\mathrm{C}=\mathrm{O}$ stretching modes (amide I), the peak at $1540 \mathrm{~cm}^{-1}$ is assigned to $\mathrm{N}-\mathrm{H}$

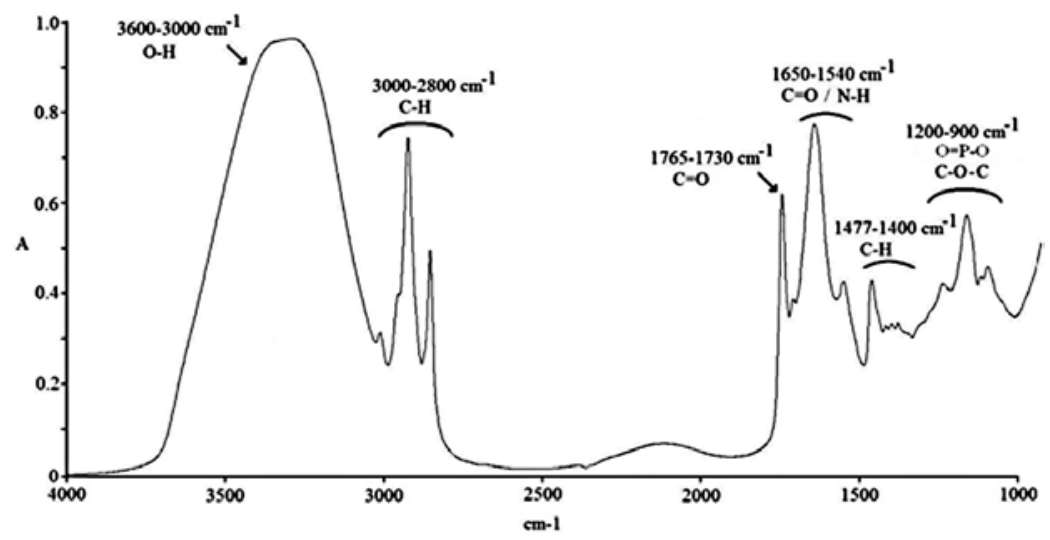

Fig. 1. Mid-infrared spectra of pure milk 
bending and C-N stretching vibrations (amide II). Lipids are present at $1477-1400 \mathrm{~cm}^{-1}$ (C-H stretch vibrations). The band at $1100 \mathrm{~cm}^{-1}$ corresponds to $\mathrm{O}=\mathrm{P}-\mathrm{O}$ (phosphate group) of the casein proteins. Mono- and polysaccharide groups are present at $1200-900 \mathrm{~cm}^{-1}$ (C-O-C stretch bonds related to lactose) (Fig. 1).

Spectra of milk samples spiked with TCs

Figure 2 shows the spectra of milk with diverse concentrations of tetracycline $(10-400 \mu \mathrm{g} / 1)$.

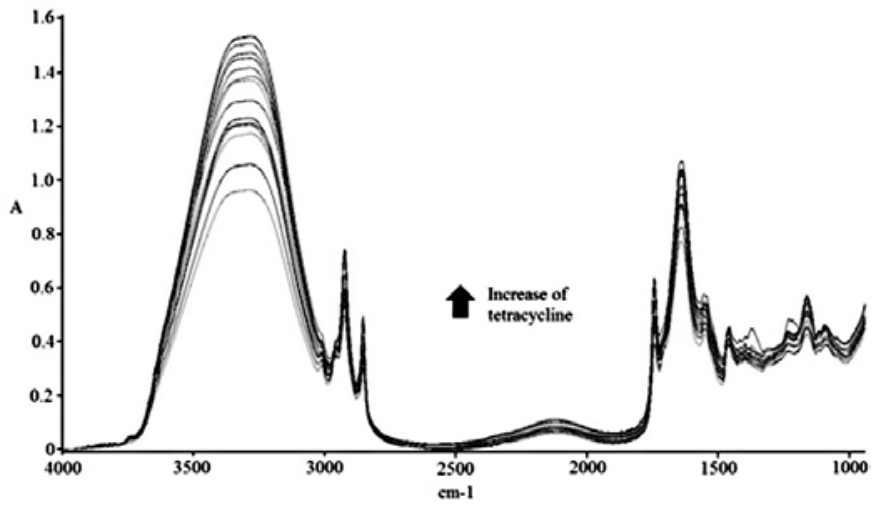

Fig. 2. Mid-infrared spectra of milk with different concentrations of tetrac

\section{Chemometric models for the prediction of TCs concentrations}

Table 1 illustrates the statistics of chemometric models for the predictions of tetracycline, chlortetracycline, and oxytetracycline in milk. The highest $\mathrm{R}^{2}$ and the lowest SEC were analysed to choose the best models (Table 1).

Table 1. Calibration and validation data of the models developed with Partial Components Regression (PCR) and Partial Least Squares (PLS1 and PLS2) algorithms to predict the concentration of tetracycline residues in milk.

\begin{tabular}{|c|c|c|c|c|c|c|}
\hline \multirow[b]{2}{*}{ Calibration set $^{\text {a }}$} & \multicolumn{3}{|c|}{ Calibration $(\mathrm{n}=30)$} & \multicolumn{3}{|c|}{ Validation $(\mathrm{n}=5)$} \\
\hline & Algorithm & No. of factors ${ }^{b}$ & $\mathrm{R}^{2 \mathrm{c}}$ & $\mathrm{SEC}^{\mathrm{d}}$ & $\mathrm{R}^{2 \mathrm{c}}$ & $\mathrm{SEP}^{\mathrm{e}}$ \\
\hline \multirow{3}{*}{ Milk-tetracycline } & PCR & 6 & 0.998 & 4.70 & -- & --- \\
\hline & PLS1 & 9 & 0.999 & 1.81 & 0.999 & 1.21 \\
\hline & PLS2 & 7 & 0.999 & 2.94 & -- & --- \\
\hline \multirow{3}{*}{ Milk-chlortetracycline } & PCR & 5 & 0.967 & 19.35 & -- & -- \\
\hline & PLS1 & 11 & 0.998 & 2.78 & 0.989 & 2.45 \\
\hline & PLS2 & 7 & 0.981 & 17.32 & -- & --- \\
\hline \multirow{3}{*}{ Milk-oxytetracycline } & PCR & 6 & 0.965 & 22.73 & -- & --- \\
\hline & PLS1 & 6 & 0.997 & 2.95 & 0.998 & 2.93 \\
\hline & PLS2 & 9 & 0.994 & 9.66 & -- & --- \\
\hline
\end{tabular}

${ }^{a}$ Calibration set were prepared ranging from $10-400 \mu \mathrm{g} / 1$

a Optimal number of factors

${ }^{\mathrm{c}}$ Coefficient of determination $\left(\mathrm{R}^{2}\right)$ should be as close to 1 as possible

d Standard Error of Calibration (SEC) should be as low as possible

e Standard Error of Prediction (SEP) should be as low as possible

Note: Best model shown in bold type 
Statistical results of the calibration models developed with the three algorithms (PLS1, PLS2 and PCR) to predict tetracycline, chlortetracycline, and oxytetracycline concentrations in milk revealed values of $\mathrm{R}^{2}$ of: $0.999,0.998$, and 0.997 , respectively, for the PLS1 algorithm and were higher than 0.998, 0.967, and 0.965, respectively, for the PCR and 0.999, 0.981, and 0.994, respectively, for the PLS2 algorithm (Table 1).

The SEC values evaluate the goodness of fit of the regression during calibration. The SEC was within the range of 1.81-2.95 for PLS1 model and between 4.70 and 22.73 for PCR model and 2.94-17.32 for the PLS2 model (Table 1).

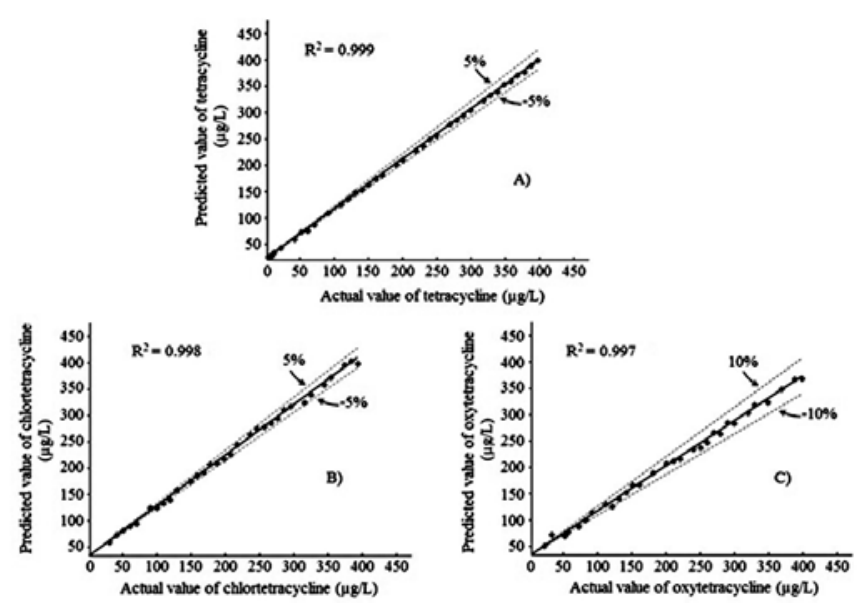

Fig. 3. Plots of predicted values versus actual values of milk with: A) tetracycline, B) chlortetracycline, and C) oxytetracycline of calibration samples determined by the Partial Least Square 1 algorithm

Figure 3 shows the data between expected values and real values by PLS1 algorithm for tetracycline, chlortetracycline, and oxytetracycline, respectively. Figure 3 depicts the good prediction ability of the models; the dashed lines in Fig. 3 illustrate the \% RD (percentage relative difference). Excellent correlations were also obtained for tetracycline, chlortetracycline, and oxytetracycline concentrations since \% RD values were less than $\pm 5 \%$ for tetracycline and chlortetracycline, whereas $\% \mathrm{RD}$ values were less than $\pm 10 \%$ for oxytetracycline.

The PLS1 models were applied to the validation set of fifteen external samples (five of each antibiotic). Table 1 describes SEP and $\mathrm{R}^{2}$ between predicted and real values of the validation set. $\mathrm{R}^{2}$ between the predicted and real values of the validation set was higher than 0.9 (tetracycline, $\mathrm{R}^{2}=0.999$; chlortetracycline, $\mathrm{R}^{2}=0.989$ and oxytetracycline, $\mathrm{R}^{2}=0.998$ ) (Table 1). The results of Table 1 indicate good prediction according to Tamaki and Mazza (2011). The SEP values specify the ability of the model to predict unknown samples. Table 1 shows the SEP values in the range of 1.21-2.93.

Table 2 shows the correlation between predicted values and real values for each antibiotic (tetracycline, chlortetracycline, and oxytetracycline) in the validation set. The statistical parameters (Mahalanobis distance and residual error) were less than 0.75 and 2.03, respectively (Table 2 ).

Classification of pure milk and its mixtures with TCs (milk-tetracycline, milkchlortetracycline and milk-oxytetracycline) using SIMCA.

Figure 4 (Plate VII) shows the 3D-PCA scores plot of the populations derived from 
Table 2. External validation data of the chemometric model developed with Partial Least Squares (PLS1) algorithm.

\begin{tabular}{lcccc}
\hline Validation set $(\mathrm{n}=5)$ & Actual value $(\mu \mathrm{g} / \mathrm{l})$ & Predicted value $(\mu \mathrm{g} / \mathrm{l})$ & Mahalanobis distance $^{\mathrm{a}}$ & Residual error $^{\mathrm{b}}$ \\
\hline \multirow{3}{*}{ Milk-tetracycline } & 30 & 30.73 & 0.34 & 1.58 \\
& 80 & 81.58 & 0.56 & 1.78 \\
& 180 & 178.26 & 0.41 & 1.40 \\
& 260 & 260.61 & 0.37 & 1.04 \\
Milk-chlortetracycline & 310 & 311.20 & 0.46 & 1.81 \\
& 80 & 78.37 & 0.54 & 1.65 \\
& 140 & 137.70 & 0.18 & 1.26 \\
& 230 & 226.81 & 0.34 & 2.03 \\
Milk-oxytetracycline & 310 & 315.92 & 0.45 & 1.63 \\
& 370 & 372.50 & 0.54 & 1.85 \\
& 40 & 43.29 & 0.75 & 1.86 \\
& 170 & 171.32 & 0.25 & 1.79 \\
& 230 & 225.65 & 0.24 & 1.32 \\
& 310 & 316.47 & 0.69 & 1.64 \\
& 360 & 357.76 & 0.39 & 1.28 \\
\hline
\end{tabular}

${ }^{a}$ Mahalanobis distance should be as low as possible, not to exceed 1

${ }^{\mathrm{b}}$ Residual error should be as low as possible, not to exceed 3

Table 3. Interclass distances of the different populations derived from Soft Independent Modeling of Class Analogy model.

\begin{tabular}{lcccc}
\hline Class & Milk & Milk-tetracycline & Milk-chlortetracycline & Milk-oxytetracycline \\
\hline Milk & 0 & 11.51 & 9.93 & 8.31 \\
Milk-tetracycline & & 0 & 6.61 & 7.62 \\
Milk-chlortetracycline & & & 0 & 7.44 \\
Milk-oxytetracycline & & & & 0 \\
\hline
\end{tabular}

Note: Interclass distances should be as high as possible, minimum 3

SIMCA (pure milk, milk-tetracycline, milk-chlortetracycline and milk-oxytetracycline). The box around each group indicates the confidence level (99\%), and the points in the group signify the spectrum of each sample (pure milk, milk-tetracycline, milk-chlortetracycline and milk-oxytetracycline).

Table 3 presents the interclass distances between the groups (pure milk, milk-tetracycline, milk-chlortetracycline and milk-oxytetracycline). These results show that the groups have interclass distances higher than 3.0, which indicates that the SIMCA model is able to separate pure milk samples and milk samples with antibiotics.

The accuracy of the SIMCA model was determined by analysing the set of external validation samples ( 5 additional spectra of each class). Table 4 shows the results of the validation set. As can be seen, the samples were identified appropriately. The statistical indicators (total distance ratio and residual distance) are within the allowed range (Table 4). The results indicate that the SIMCA model can distinguish pure milk, milk-tetracycline, milk-chlortetracycline and milk-oxytetracycline with an excellent confidence limit (99\%).

\section{Discussion}

The difference in the infrared energy absorption is reflected by the different intensities of the spectra for each sample. A spectrum for a sample with a higher antibiotic content 
Table 4. External validation data of the Soft Independent Modeling of Class Analogy model.

\begin{tabular}{cccccc}
\hline Samples & Specified material $^{\text {a }}$ & Identified material $^{\mathrm{b}}$ & Result $^{\mathrm{c}}$ & Total distance ratio $^{\mathrm{d}}$ & Residual distance $^{\mathrm{e}}$ \\
\hline $1-5$ & Milk & Milk & Identified & $0.57-0.86$ & $0.78-1.17$ \\
$1-5$ & Milk-tetracycline & Milk-tetracycline & Identified & $0.54-0.79$ & $0.79-1.15$ \\
$1-5$ & Milk-chlortetracycline & Milk-chlortetracycline & Identified & $0.74-0.93$ & $1.01-1.25$ \\
$1-5$ & Milk-oxytetracycline & Milk-oxytetracycline & Identified & $0.27-0.51$ & $0.38-0.72$ \\
\hline
\end{tabular}

a Specified material, the material indicated during validation

${ }^{\mathrm{b}}$ Identified material, the material identified during validation

${ }^{\mathrm{c}}$ Result, the material identified or not as the specified material

d Total distance ratio must be less than 1.0 for a sample to be classified

${ }^{\mathrm{e}}$ Residual distance should be as low as possible

has a higher absorbance. Hence, the spectra of Fig. 2 revealed modifications in the Mid FTIR region due to the variation in levels of the functional groups. Same changes in the absorbance were observed with the other antibiotics (chlortetracycline and oxytetracycline).

The calibration models developed with PLS1 algorithm show the best results for predicting the concentrations of these antibiotics because the statistics are considered good prediction according to Tamaki and Mazza (2011). Also, the PLS1 algorithm has lower SEC values and higher $\mathrm{R}^{2}$ compared to the PCR and PLS2 models; the above mentioned indicates that the PLS1 model has better calibration power than the PCR and PLS2 models. This is revealed in the validation set. According to the above mentioned results, the PLS1 algorithm can predict tetracycline, chlortetracycline, and oxytetracycline concentrations in milk samples.

It is worth mentioning that the limit of detection (LOD) of the calibration models to predict tetracycline, chlortetracycline and oxytetracycline concentrations in milk is above $10 \mu \mathrm{g} / \mathrm{l}(\mathrm{LOD}>10 \mu \mathrm{g} / \mathrm{l})$. In general, the LODs of the developed chemometric models are acceptable because they are below the MRLs established by the FDA $(300 \mu \mathrm{g} / \mathrm{l})$, the European Union and the Codex Alimentarius Commission (100 $\mu \mathrm{g} / \mathrm{l})$ (Commission Regulation 37/2010). Also, this LOD $(>10 \mu \mathrm{g} / \mathrm{l})$ is in agreement with the limits of detection reported by other authors that use complex methodologies such as thin layer chromatography $(50-300 \mu \mathrm{g} / \mathrm{l})$; capillary electrophoresis $(2-9 \mu \mathrm{g} / \mathrm{l})$ and biosensors $(5-25$ $\mu \mathrm{g} / \mathrm{l})(\mathrm{Oka}$ et al. 2000; Cháfer-Pericás et al. 2010; Pérez-Fernández et al. 2012).

The SIMCA model provides interclass distances between groups (pure milk, milktetracycline, milk-chlortetracycline and milk-oxytetracycline) therefore, SIMCA's interclass distance describes quantitatively the similarity or dissimilarity of the different classes, being generally accepted that samples can be differentiated when interclass distance $>3.0$ (Graso et al. 2011). The above mentioned indicates the SIMCA model showed good group separation making it possible to distinguish between pure milk samples and milk samples with tetracycline residues. This is confirmed in the validation set. It is noteworthy that the SIMCA model has never been used to discriminate tetracycline residues in cow's milk from vibrational spectral data.

In conclusion, Mid-FTIR and chemometric analysis were able to detect and quantify tetracycline, chlortetracycline, and oxytetracycline concentrations in cow's milk at a low concentration (LOD $>10 \mu \mathrm{g} / \mathrm{l}$ ). Therefore, this technique can be used to verify the safety of milk rapidly and reliably. Further studies are needed to identify other contaminants of similar chemical structures to avoid cross reactivities. This detection technique is starting to be employed in detecting veterinary residues; therefore, these types of techniques may be used to analyse a number of foods. 


\section{Acknowledgements}

The financial support from the Consejo Nacional de Ciencia y Tecnología (CONACyT) and the Secretaría de Estudios de Posgrado e Investigación del Instituto Politécnico Nacional de México (SIP-IPN) is greatly appreciated.

\section{References}

Aderson CR, Rupp HS, Wu WH 2005: Complexities in tetracycline analysis-chemistry, matrix extraction, cleanup, and liquid chromatography. J Chromatogr A 1075: 23-32

Andersen WC, Roybal JE, Gonzales SA, Turnipseed SB, Pfenning AP Kuck LR 2005: Determination of tetracycline residues in shrimp and whole milk using liquid chromatography with ultraviolet detection and residue confirmation by mass spectrometry. Anal Chim Acta 529: 145-150

Boeckman S, Carlson KR 2006: Milk and Dairy Beef Residue Prevention Protocol. Agri-education, Inc., Stratford, IA

Casella IG, Picerno F 2009: Determination of tetracycline residues by liquid chromatography coupled with electrochemical detector and solid phase extraction. J Agric Food Chem 57: 8735-8741

Cháfer-Pericás C, Maquieira Á, Puchades R 2010: Fast screening methods to detect antibiotic residues in food samples. TrAC Trends Anal Chem 29: 1038-1049

Commission Regulation (EU) No. 37/2010. Official Journal of the European Union. L15, 1

Debuf Y 1998: The Veterinary Formulary. Pharmaceutical Press, London, 97 p.

Dračková M, Navrátilová P, Hadra L, Vorlová L, Hudcová L 2009: Determination residues of penicillin G and cloxacillin in raw cow milk using Fourier Transform Near Infrared Spectroscopy. Acta Vet Brno 78: 685-690

Feng-Jiao L, Shun-Xing L, Feng-Ying Z, Xu-Guang H, Yue-Gang Z, Teng-Xiu T, Xue-Qing W 2014: Risk assessment of nitrate and oxytetracycline addition on coastal ecosystem functions. Aquatic Toxicol 146: 76-81

Fritz JW, Zuo Y 2007: Simultaneous determination of tetracycline, oxytetracycline and 4-epitetracycline in milk by high-performance liquid chromatography. Food Chem 105: 1297-1301

Grasso EM, Yousef AE, De Lamo Castellvi S, Rodriguez-Saona LE 2011: Rapid detection and differentiation of Alicyclobacillus species in fruit juice using hydrophobic grid membranes and attenuated total reflectance infrared microspectroscopy. J Agric Food Chem 57: 10670-10674

Ibarra IS, Rodriguez JA, Miranda JM, Vega M, Barrado E 2011: Magnetic solid phase extraction based on phenyl silica adsorbent for the determination of tetracyclines in milk samples by capillary electrophoresis. J Chromatogr A 1218: 2196-2202

Meza-Márquez OG, Gallardo-Velázquez T, Dorantes-Álvarez L, Osorio-Revilla G, De la Rosa JL 2011: FT-MIR and Raman spectroscopy coupled to multivariate analysis for the detection of clenbuterol in murine model. Analyst 136: 3355-3365

Meza-Márquez OG, Gallardo-Velázquez T, Osorio-Revilla G, Dorantes-Álvarez L 2012: Detection of clenbuterol in beef meat, liver and kidney by Mid-Infrared spectroscopy (FT-Mid IR) and multivariate analysis. Inter J Food Sci Technol 47: 2342-2351

Nagel OG, Molina MP, Althaus RL 2011: Optimization of bioassay for tetracycline detection in milk by means of chemometric techniques. Letters App Microbiol 52: 245-252

Oka H, Ito Y, Matsumoto H 2000: Chromatographic analysis of tetracycline antibiotics in foods. J Chromatogr A 882: 109-133

Pena A, Lino CM, Alonso R, Barcelò D 2007: Determination of tetracycline antibiotics in edible swine tissues by liquid chromatography with spectrofluorometric detection and confirmation by mass spectrometry. J Agric Food Chem 55: 4973-4979

Pena A, Pelantova N, Lino CM, Silveira MIN, Solich P 2005: Validation of an analytical methodology for determination of oxytetracycline and tetracycline residues in honey by HPLC with fluorescence detection. J Agric Food Chem 53: 3784-3788

Pérez-Fernández V, Domínguez-Vega E, Crego AL, García MÁ, Marina ML 2012: Recent advances in the analysis of antibiotics by CE and CEC. Electrophoresis 33: 127-146

Riviere JE, Papich MG, Lees P, Elliott J, Clarke C, Anadón A, Baggot D, Brown SA, Burka JF, Clarke CR, Craigmill A 2003: USP veterinary pharmaceutical information monographs-antibiotics. J Vet Pharma Therap 26: $v-271$

Schneider MJ, Braden SE, Reyes-Herrera I, Donoghue DJ 2007: Simultaneous determination of fluoroquinolones and tetracyclines in chicken muscle using HPLC with fluorescence detection. J Chromatogr B 846: 8-13

Sivakesava S, Irudayaraj J 2002: Rapid determination of Tetracycline in milk by FT-MIR and FT-NIR Spectroscopy. J Dairy Sci 85: 487-493

Tamaki Y, Mazza G 2011: Rapid determination of lignin content of straw using attenuated total reflectance Fourier transform mid-infrared spectroscopy. J Agric Food Chem 59: 504-512

Toldrá F, Reig M 2006: Methods for rapid detection of chemical and veterinary drug residues in animal foods. Trends Food Sci Technol 17: 482-489

Viñas P, Balsalobre N, López-Erroz C, Hernández-Córdoba M 2004: Liquid chromatography with ultraviolet absorbance detection for the analysis of tetracycline residues in honey. J Chromatogr A 1022: 125-129

Yildiz Ö, Unluturk S 2009: Differential scanning calorimetry as a tool to detect antibiotic residues in ultra high temperature whole milk. Inter J Food Sci Technol 44: 2577-2582 


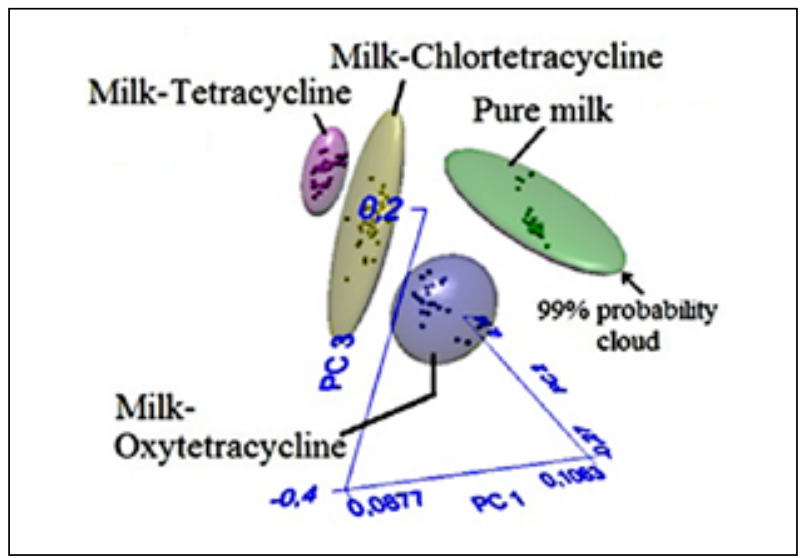

Fig. 4. Three-dimensional principal component analysis scores plot of the populations derived from Soft Independent Modeling of Class Analogy model: pure milk, milk-tetracycline, milk-chlortetracycline and milkoxytetracycline 